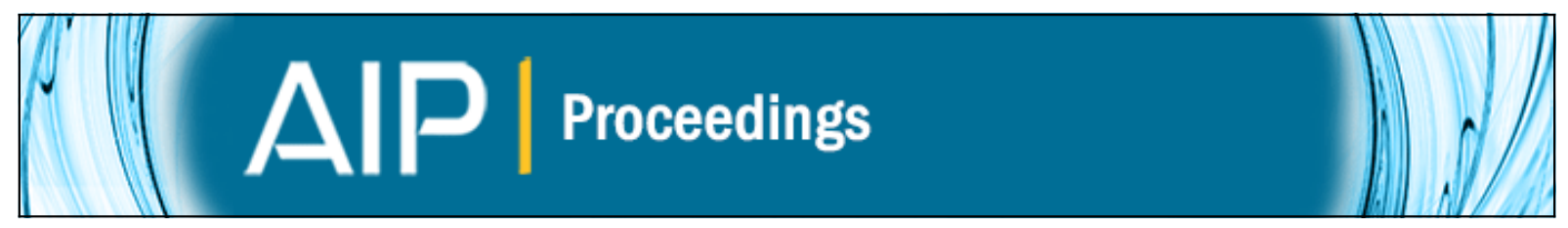

\title{
Monte Carlo modeling of electron density in hypersonic rarefied gas flows
}

Jin Fan, Yuhuai Zhang, and Jianzheng Jiang

Citation: AIP Conference Proceedings 1628, 148 (2014); doi: 10.1063/1.4902586

View online: http://dx.doi.org/10.1063/1.4902586

View Table of Contents: http://scitation.aip.org/content/aip/proceeding/aipcp/1628?ver=pdfcov

Published by the AIP Publishing

Articles you may be interested in

Modeling study of rarefied gas effects on hypersonic reacting stagnation flows

AIP Conf. Proc. 1628, 1310 (2014); 10.1063/1.4902742

Monte Carlo Simulation of Hypersonic Rarefied Gas Flow using Rotationally and Vibrationally Inelastic Cross Section Models

AIP Conf. Proc. 1333, 1245 (2011); 10.1063/1.3562814

Variable Sphere Molecular Model in the Monte Carlo Simulation of Rarefied Gas Flow

AIP Conf. Proc. 663, 358 (2003); 10.1063/1.1581570

DSMC simulation with gas-surface interaction models in hypersonic rarefied flow

AIP Conf. Proc. 585, 331 (2001); 10.1063/1.1407579

Monte Carlo analysis of macroscopic fluctuations in a rarefied hypersonic flow around a cylinder

Phys. Fluids 12, 1226 (2000); 10.1063/1.870372 


\title{
Monte Carlo Modeling of Electron Density in Hypersonic Rarefied Gas Flows
}

\author{
Jin Fan, Yuhuai Zhang and Jianzheng Jiang \\ State Key Laboratory of High Temperature Gas Dynamics, \\ Institute of Mechanics, Chinese Academy of Sciences, Beijing 100190, China
}

\begin{abstract}
The electron density distribution around a vehicle employed in the RAM-C II flight test is calculated with the DSMC method. To resolve the mole fraction of electrons which is several orders lower than those of the primary species in the free stream, an algorithm named as trace species separation (TSS) is utilized. The TSS algorithm solves the primary and trace species separately, which is similar to the DSMC overlay techniques; however it generates new simulated molecules of trace species, such as ions and electrons in each cell, basing on the ionization and recombination rates directly, which differs from the DSMC overlay techniques based on probabilistic models. The electron density distributions computed by TSS agree well with the flight data measured in the RAM-C II test along a decent trajectory at three altitudes $81 \mathrm{~km}, 76 \mathrm{~km}$, and $71 \mathrm{~km}$.
\end{abstract}

Keywords: hypersonic rarefied gas flow, electron density, DSMC, TSS, RAM-C II flight test, PACS: $47.70 \mathrm{Nd}, 34.50 . \mathrm{Gb}$

\section{INTRODUCTION}

The DSMC method is a powerful tool to analyze various rarefied gas flows [1], and it is widely used in the context of aerospace engineering [2,3]. An important but not completely solved yet issue is how to predict electron density in hypersonic rarefied gas flows using DSMC. The problem was firstly encountered by Bird [4] in 1989 when calculating the electron density distribution under the RAM-C II flight test condition at altitude $81 \mathrm{~km}$. The Radio Attenuation Measurement (RAM) experiments involved a series of hypersonic reentry flights carried out by the United States Air Force in 1960's, and the RAM-C II was one of them [5,6]. As finally remarked in Ref. [4], the comparison between the DSMC and measured results showed that "the degree of agreement is only just outside what might be called the 'acceptable limits', and additional test cases appear to be required to explain the discrepancy."

Many efforts have been devoted to the issue since then, and those from Boyd and his collaborators were mostly lasting \& systematic. The basic treatment in their studies was to assign different ratios of simulated to real molecules for different species, with the help of a new weighting scheme that explicitly conserves both linear momentum and energy during collisions [7]. However, the problem seems not solved completely because certain artificial treatments are still unable to be avoided, e.g., in Ref. [8], the ionizing reaction probabilities artificially increased by a factor of 30 to increase the number of charged particles in the DSMC computation by a factor of about 120 .

The present paper will employ an algorithm named as trace species separation (TSS) to calculate the electron density distribution in a hypersonic rarefied gas flow. The TSS algorithm [9] solves primary and trace species separately, which is similar to the DSMC overlay techniques [10-12]; however it generates the new simulated molecules of trace species, such as ions and electrons, basing on the ionization and recombination rates directly. By comparison, the DSMC overlay techniques are based on a probabilistic model. In the following sections, we will firstly describe the TSS algorithm; then apply it to the RAM-C II flight test conditions at typical altitudes (81km, $76 \mathrm{~km}$, and $71 \mathrm{~km}$ ), and compare the TSS results to the measured data reported in Refs. [5, 6].

\section{THE TSS ALGORITHM}

\section{Primary Species}

The primary species in the free stream past a hypersonic vehicle consist of $\mathrm{N}_{2}$ and $\mathrm{O}_{2}$. After a strong shock wave, part of them are heated and converted into $\mathrm{N}, \mathrm{O}$ and $\mathrm{NO}$ via dissociation and exchange reactions. For the five species $\left(\mathrm{N}_{2}, \mathrm{O}_{2}, \mathrm{NO}, \mathrm{N} \& \mathrm{O}\right)$, a frequently used model is compiled by Bortner [13], and includes 15 dissociation reactions and 4 exchange reactions. The DSMC calculations of these species in hypersonic rarefied gas flows,

Proceedings of the 29th International Symposium on Rarefied Gas Dynamics

AIP Conf. Proc. 1628, 148-154 (2014); doi: 10.1063/1.4902586

(C) 2014 AIP Publishing LLC 978-0-7354-1265-1/\$30.00 
though complicated due to the rotational and vibrational energy excitations as well as chemical reactions, are an issue well studied and solved [1]. The present paper employs several widely used models [1], such as the VHS model, the Larsen-Borgnakke model, and the total collision energy model. The implementation of these models in a code named as 3R was validated and reported in Refs. [14-17].

\section{Trace Species}

There are a number of trace species in air. What we need to consider is only those playing a key role. For instance, in the RAM-C II flight test, the electron density mainly depended on the ionization reactions between nitrogen and oxygen atoms, as well as the backward recombination reactions. Both of them are given in Table 1, together with the reaction rates recommended by Park [18], where $T_{a}=T_{v}^{q} \times T_{t-r}^{1-q}, T_{v}$ is the vibrational temperature, $T_{t}$ the translational and rotational temperature, and the parameter $q$ is a constant between 0.3 and 0.5 .

The TSS algorithm to compute trace species such as $\mathrm{NO}^{+}, \mathrm{N}_{2}^{+}, \mathrm{O}_{2}^{+}$and $e$ consists of the following steps.

1) Generation of ions and electrons

Within each time step $\Delta t$, the number of new simulated ions in the $j$-th cell is decided by the forward and backward reactions jointly, e.g.

with

$$
N_{j, N O^{+}}=N_{j, N O^{+}}^{F}-N_{j, N O^{+}}^{B}
$$

$$
\begin{aligned}
& N_{N O^{+}, j}^{F}=k_{N-O} n_{j}^{N} n_{j}^{O} \Delta V_{j} \Delta t / \gamma_{t}, \\
& N_{N O^{+}, j}^{B}=k_{N O^{+}-e} n_{j}^{N O+} n_{j}^{e} \Delta V_{j} \Delta t / \gamma_{t},
\end{aligned}
$$

where $k_{\mathrm{N}-\mathrm{O}}$ is the ionization reaction rate between $\mathrm{N}$ and $\mathrm{O}$ given in Table $1 ; k_{\mathrm{NO}^{+}-e}$ the backward recombination rate, $n_{j}^{N}$ and $n_{j}^{O}$ the number densities of $\mathrm{N}$ and $\mathrm{O}$ in the cell, respectively, obtained in the first round of TSS; $n_{j}^{N O+}$ and $n_{j}^{e}$ the number densities of $\mathrm{NO}^{+}$and electron in the cell updated every time step during the second round of TSS until they reach a steady state; $\Delta V_{j}$ the cell volume, and $\gamma_{t}$ the ratio of real-to-simulated particles of trace species.

The translational, rotational and vibtational energies of a new ion are initialized based on the corresponding temperatures of the cells within which it is generated. The implementation is the same as the initialization of simulated molecules from a known state that is usually the first step to start DSMC calculations.

Compared with the DSMC overlay techniques [10-12], the generation of new simulated particles of trace species in the TSS algorithm such as $\mathrm{NO}^{+}$and electrons by Eqs. (1)-(3) is more convenient and efficient. A query may arise whether it works when the collisions become so rare, e.g. for a spacecraft glow over an altitude range of $140-180 \mathrm{~km}$ studied in [10-12]. The essence of the query is whether the concept of reaction rate is applicable to free molecular flows that are usually defined according to the value of $K n$, e.g., $K n>10$ or 100 . The answer should be affirmative; however, the temperature model perhaps needs certain modifications in consistence with extreme nonequilibrium due to rare collisions. For the present situation which is far from free molecules, it is proper to directly apply the reaction rates given in Table 1 to compute the generation rates for the ions and electrons.

TABLE 1. Reaction rate coefficients for associative ionization and recombination reactions

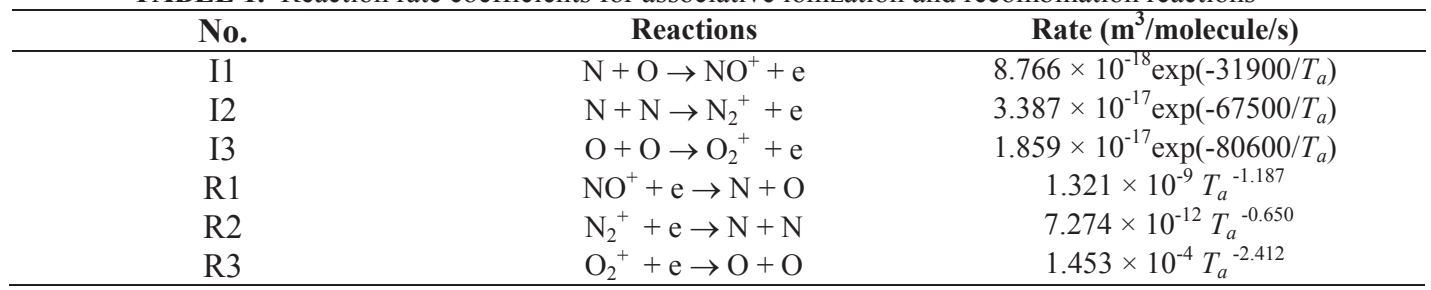


2) Movement of ions and electrons

After a time step, the movement of ion $j$ is simply as:

$$
\mathrm{r}_{\mathrm{j}, \mathrm{i}}(\mathrm{t}+\Delta \mathrm{t})=\mathrm{r}_{\mathrm{j}, \mathrm{i}}(\mathrm{t})+\mathrm{v}_{\mathrm{j}, \mathrm{i}} \times \Delta \mathrm{t}
$$

where $v_{j, i}$ is its velocity.

All electrons move together with the corresponding ions. Such a quasi-neutrality simplification is reasonable because the ratio of the Debye length, $\lambda_{\mathrm{D}}$, to the free mean path of air molecules, $\lambda_{\text {air }}$, under the RAM-C II test conditions is very small.

$$
\frac{\lambda_{\mathrm{D}}}{\lambda_{\text {air }}} \sim\left(\frac{\varepsilon_{0} \mathrm{kT}_{\mathrm{e}}}{\mathrm{n}_{\mathrm{e}} \mathrm{e}^{2}}\right)^{0.5} \times \frac{1}{\mathrm{n}_{\text {air }} \sigma_{\mathrm{T}}}=\alpha_{0} \sqrt{\mathrm{T}_{\mathrm{e}} / \mathrm{n}_{\mathrm{e}}} / \mathrm{n}_{\text {air }},
$$

where $\alpha_{0}=\sqrt{\varepsilon_{0} k} /\left(e \sigma_{T}\right) \sim 10^{20} ; n_{\text {air }}$ is the free stream number density whose order of magnitude is approximately $10^{20} \mathrm{~m}^{-3}$ around altitude $80 \mathrm{~km} ; n_{\mathrm{e}}$ the electron number density with an order of magnitude between $10^{15} \sim 10^{18} \mathrm{~m}^{-3}$; and the electron temperature $T_{\mathrm{e}} \sim 10,000 \mathrm{~K}$. Substituting these values into Eq. (5) leads to

$$
\lambda_{\mathrm{D}} / \lambda_{\text {air }} \sim 10^{-5}-10^{-7} .
$$

Since the cell size is comparable to $\lambda_{\text {air }}$, the assumption of electric neutrality is well satisfied within every cell, and it allows the electrons to move along with the corresponding ions.

3) Ion collisions

Since the mole fractions of $\mathrm{NO}^{+}, \mathrm{N}_{2}{ }^{+}$, and $\mathrm{O}_{2}{ }^{+}$are much lower than those of primary species, collisions with ions mainly come from the particles of the primary neutral species. For example, the number of collisions between $\mathrm{NO}^{+}$ and the particles of primary species $i$ in cell $j$ each time step is:

$$
N_{c, i}^{N O^{+}}=n_{i}^{N O^{+}} n_{i}^{j} \overline{\sigma_{T} c_{r}} \Delta V_{i} \Delta t
$$

The velocity, rotational and vibrational energies of a simulated particle of the primary species are generated based upon a local Maxwellian distribution.

4) Sample and average

A sampling procedure begins when the DSMC calculation of trace species reaches a steady state, e.g. the number density for $\mathrm{NO}^{+}$is:

$$
n_{i}^{N O^{+}}=\frac{\gamma_{t}}{N_{S} \Delta V_{i}} \sum_{\ell=1}^{N_{S}} N_{\ell, i}^{N O^{+}}
$$

where $\mathrm{N}_{\mathrm{s}}$ is the total number of sampling time steps, and $\mathrm{N}_{\ell, i}^{\mathrm{NO}}$ is the number of $\mathrm{NO}^{+}$during the $\ell$-th sampling time step. The electron density is equal to the summation over the number densities of the three ions.

\section{RESULTS AND DISCUSSIONS}

\section{Computational Conditions}

As shown in Fig. 1, the vehicle employed in the RAM-C II experiment was a blunted cone with a spherical nose, and the sphere radius was $0.1524 \mathrm{~m}$. The cone angle was $9^{\circ}$, and the total length about $1.3 \mathrm{~m}$. The reentry velocity was about $7.8 \mathrm{~km} / \mathrm{s}$ with an attack angle near $0^{\circ}$. The electron number density distributions were measured using two diagnostics, namely microwave reflectometer and Langmuir probe, at several locations in the plasma layer surrounding the vehicle along a decending trajectory from $90 \mathrm{~km}$ to $60 \mathrm{~km}[5,6]$.

An axisymmetric assumption is employed here due to the attack angle near $0^{\circ}$. The computational domain is rectangular whose sizes are properly chosen according to the simulated altitudes, which are given in Table 2, 


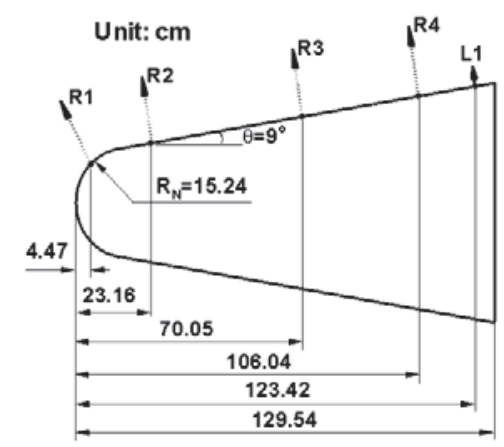

FIGURE 1. Schematic of the vehicle and measurement locations $R_{1}-R_{4}$ and $L_{1}$ in the RAM-C II test [5].

TABLE 2. Computational parameters used in the first round TSS simulations

\begin{tabular}{cccccc}
\hline Altitude & Domain & Cell & Time step & $\mathbf{N}_{\mathbf{t}}$ & CPU \\
\hline $81 \mathrm{~km}$ & $1.39 \mathrm{~m} \times 0.73 \mathrm{~m}$ & $1400 \times 200$ & $2 \times 10^{-7} \mathrm{~s}$ & $1 \times 10^{7}$ & $20 \mathrm{hr}$ \\
$76 \mathrm{~km}$ & $1.38 \mathrm{~m} \times 0.70 \mathrm{~m}$ & $1400 \times 200$ & $8 \times 10^{-8} \mathrm{~s}$ & $5 \times 10^{7}$ & $20 \mathrm{hr}$ \\
$71 \mathrm{~km}$ & $1.38 \mathrm{~m} \times 0.70 \mathrm{~m}$ & $1600 \times 200$ & $5 \times 10^{-8} \mathrm{~s}$ & $6 \times 10^{7}$ & $40 \mathrm{hr}$ \\
\hline
\end{tabular}

together with the other computational parameters, where $\mathrm{N}_{\mathrm{t}}$ denotes the total number of simulated particles, and CPU the computational time consumed in a server of DELL M910 with 64 processors at Institute of Mechanics, Chinese Academy of Sciences. The vehicle surface is assumed to be fully diffusive reflective with a temperature of $1500 \mathrm{~K}$ for the three cases.

\section{Typical Flow Field for Primary Species}

According to the TSS algorithm, the flow field of primary species is firstly computed using DSMC without taking into account trace species. Figure 2 presents the density, temperature and velocity fields at altitude $81 \mathrm{~km}$, as well as the $\mathrm{N}$ and $\mathrm{O}$ mole fraction distributions. All of them are necessary for calculating trace species in the second round simulations with the TSS algorithm. Similar to a typical reentry process, a strong bow shock wave clearly appears in front of the vehicle, and it leads to sharp increases of density, translational, rotational and vibrational temperatures. The flow is nonequilibrium, with the maximum temperature values of about $25,000 \mathrm{~K}, 12,000 \mathrm{~K}$, and $3000 \mathrm{~K}$, respectively. The high temperatures trigger the chemical reactions listed in Table 1, and they convert almost all $\mathrm{O}_{2}$ and certain amount of $\mathrm{N}_{2}$ in the free stream to $\mathrm{O}$ and $\mathrm{N}$, respectively, with the mole fractions of about 0.2 and 0.3 in the stagnant zone.

\section{Electron Density Distribution}

Figure 3 presents the electron and ions number density distributions computed with the TSS algorithm under the RAM-C II test conditions at altitude $81 \mathrm{~km}$. The main source of electron is the first ionization reaction in Table 1 , because the maximum number density of $\mathrm{NO}^{+}$is about $1 \times 10^{18} \mathrm{~m}^{-3}$ (Fig.3b), much higher than the maximum values of $\mathrm{N}_{2}^{+}$and $\mathrm{O}_{2}^{+}$of $5 \times 10^{16} \mathrm{~m}^{-3}$ (Fig.3c) and $5 \times 10^{15} \mathrm{~m}^{-3}$ (Fig.3d), respectively.

Figure 4 presents the electron number densities computed with the TSS algorithm and measured by microwave reflectometers [5] at $R_{1}, R_{2}, R_{3} \& R_{4}$ at three altitudes $81 \mathrm{~km}, 76 \mathrm{~km}$ and $71 \mathrm{~km}$. The locations $R_{1}-R_{4}$ are marked in Fig.1 (right). The TSS and measured results are generally in excellent agreement at all the measurement locations of altitude $81 \mathrm{~km}$, and at location $\mathrm{R}_{1}$ of altitudes $76 \mathrm{~km}$ and $71 \mathrm{~km}$, but not so satisfactory at the aft part of the vehicle as the altitude decreases.

Figure 5 presents the electron density distributions computed with the TSS algorithm and measured by Langmuir probes [6] at location $L_{1}$ marked in Fig.1 (right) at altitudes $81 \mathrm{~km}, 76 \mathrm{~km} \& 71 \mathrm{~km}$. The TSS results are almost at the upper limit of the range of measured data. Moreover, the present calculations illustrate that the effect of the recombination reactions become important as the altitude decreases. For example, at $81 \mathrm{~km}$, whether the recombination reactions are taken into account or not, the agreement between the TSS and experimental results is almost same, but at $71 \mathrm{~km}$, if the recombination reactions are not taken into account, the TSS results will be much higher than the experimental data. 


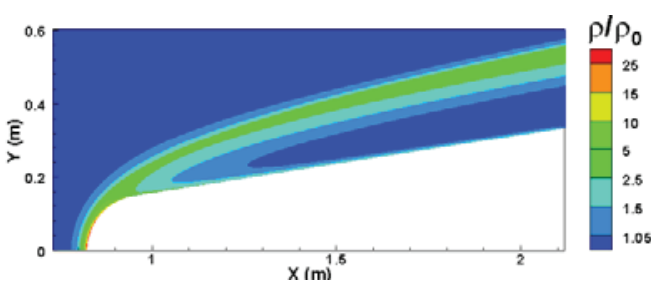

a) Normalized density

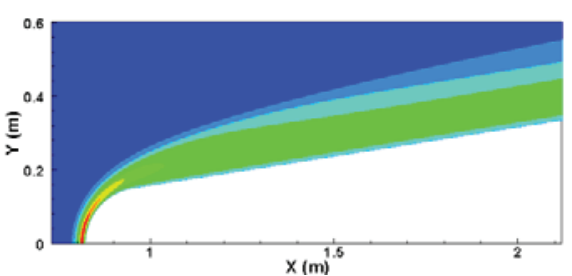

c) Rotational temperature

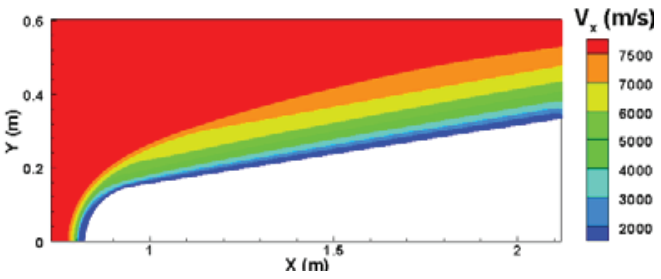

e) Velocity in $x$ direction

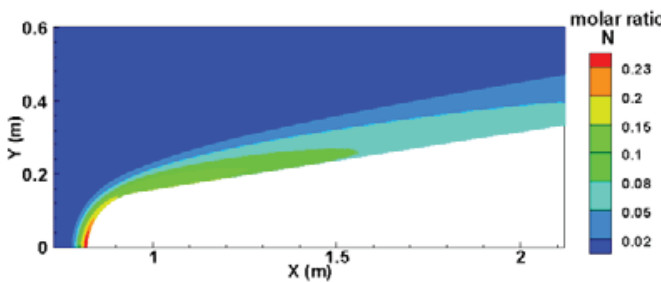

g) Mole fraction of $\mathrm{N}$

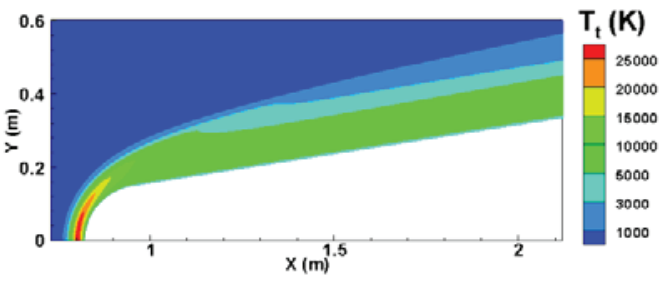

b) Translational temperature

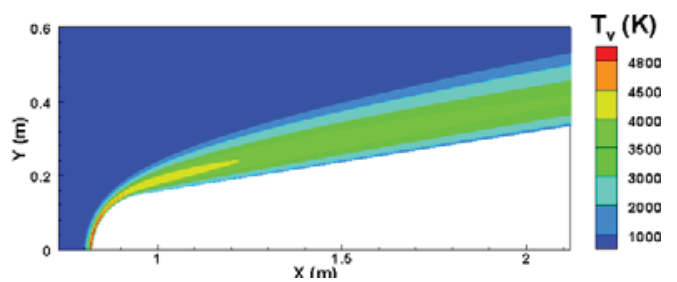

d) Vibrational temperature

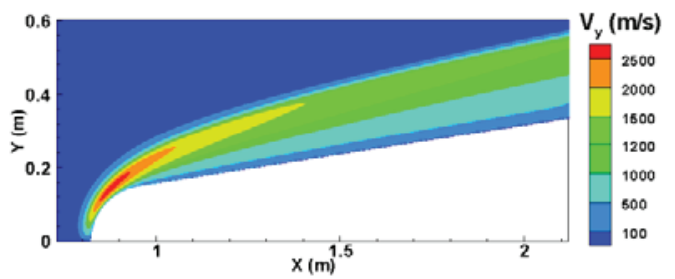

f) Velocity in y direction

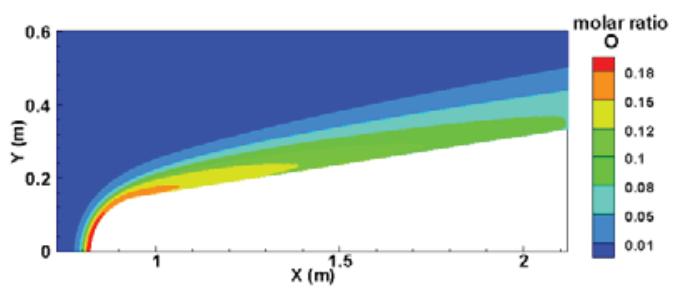

h) Mole fraction of $\mathrm{O}$

Figure 2. Flow field of primary species computed by DSMC under the RAM-C II test condition at altitude $81 \mathrm{~km}$.
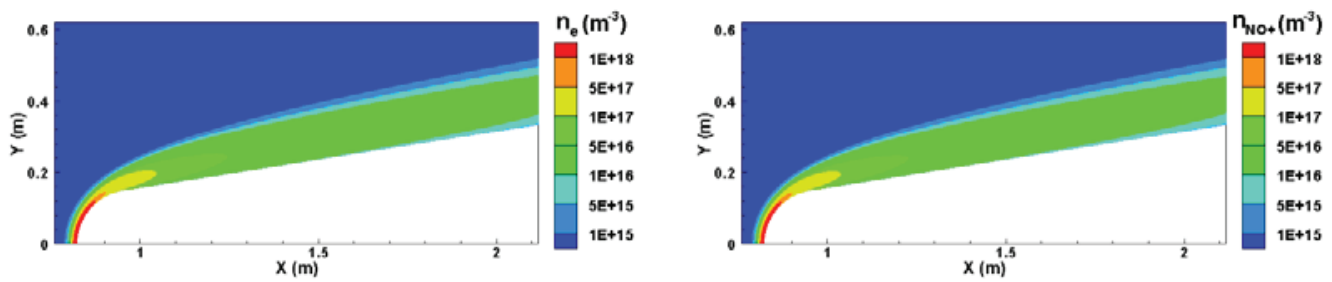

a) Electron

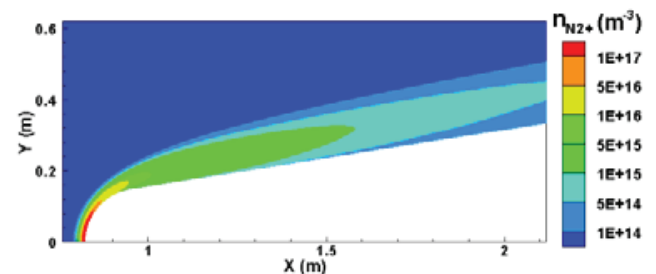

c) $\mathrm{N}_{2}^{+}$

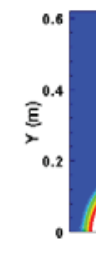

b) $\mathrm{NO}^{+}$

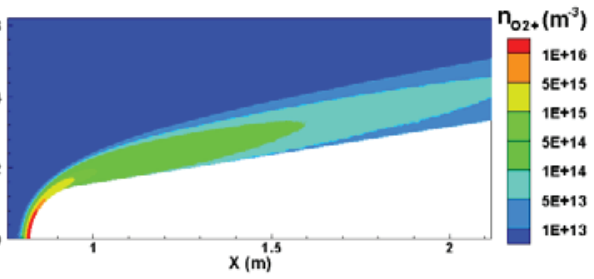

d) $\mathrm{O}_{2}^{+}$

Figure 3. Ions and electron distributions, computed by TSS under the RAM-C II test condition, altitude $81 \mathrm{~km}$. 


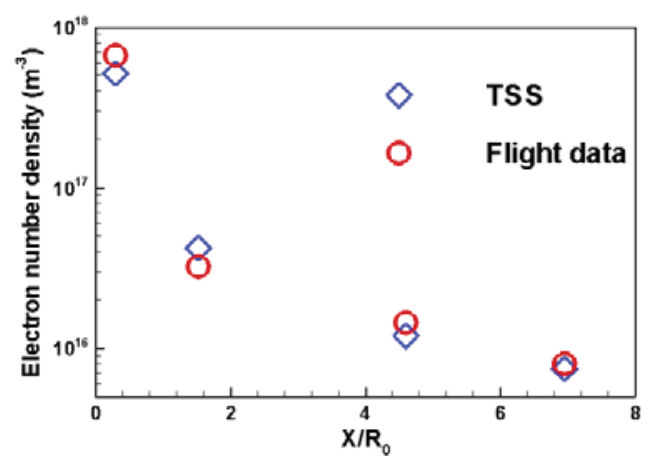

a) Altitude $81 \mathrm{~km}$

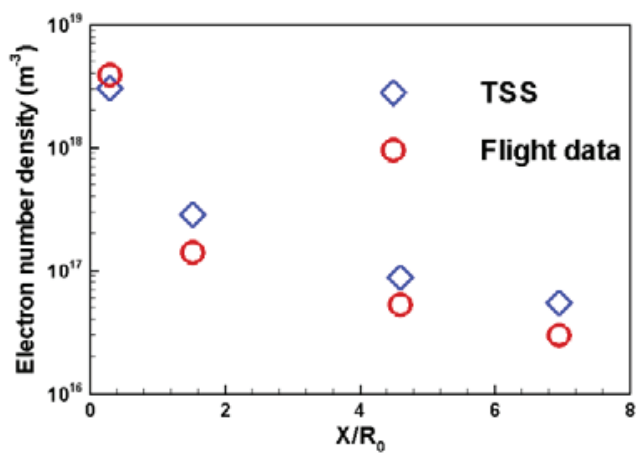

b) Altitude $76 \mathrm{~km}$

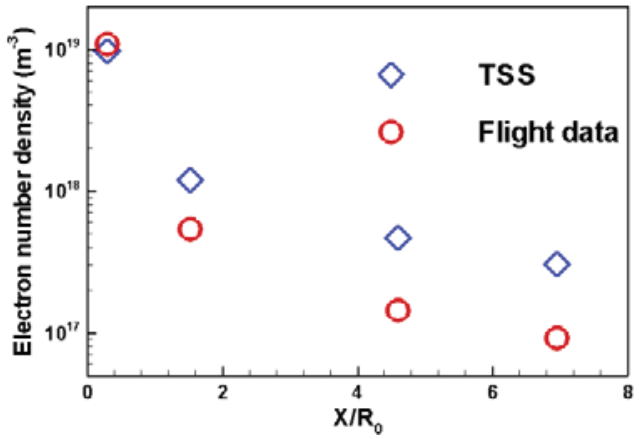

c) Altitude $71 \mathrm{~km}$

Figure 4. Maximum electron number densities at locations $\mathrm{R}_{1}, \mathrm{R}_{2}, \mathrm{R}_{3} \& \mathrm{R}_{4}$, along the decent trajectory of RAM-C II flight, at altitudes $81 \mathrm{~km}, 76 \mathrm{~km}$ and $71 \mathrm{~km}$.

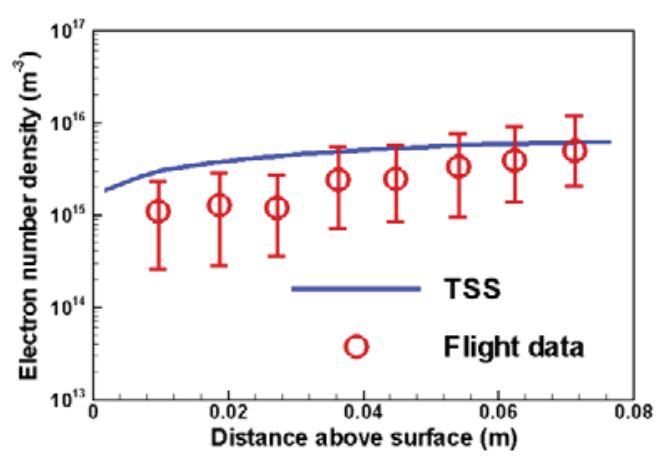

a) Altitude $81 \mathrm{~km}$

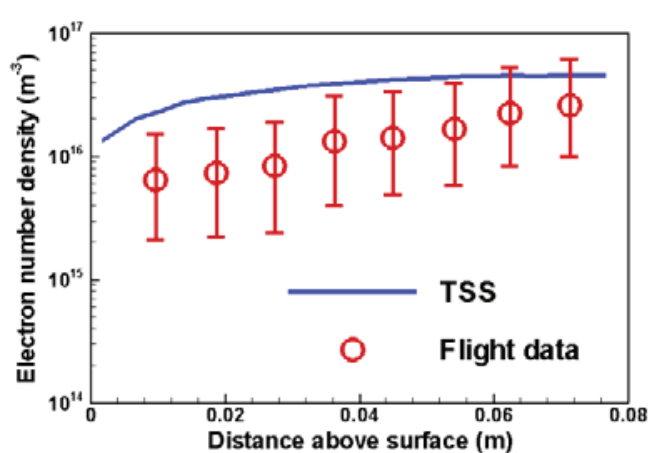

b) Altitude $76 \mathrm{~km}$

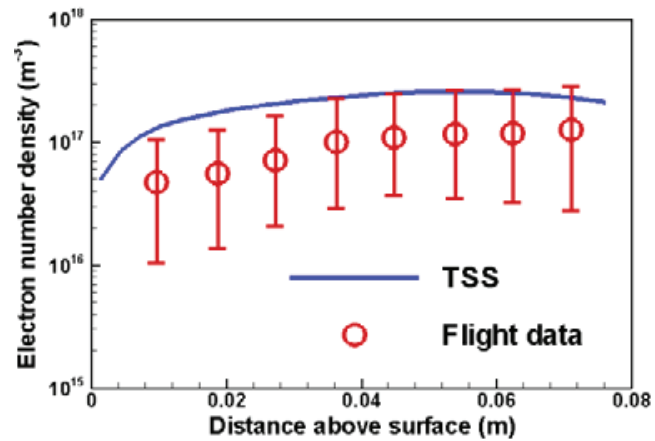

c) Altitude $71 \mathrm{~km}$

Figure 5. Electron number density at $\mathrm{L}_{1}$, along the decent trajectory of RAM-C II flight, at altitudes $81 \mathrm{~km}, 76 \mathrm{~km}$ and $71 \mathrm{~km}$. 


\section{CONCLUSIONS}

The electron density distributions under the RAM C-II flight test conditions at altitudes $81 \mathrm{~km}, 76 \mathrm{~km}$, and $71 \mathrm{~km}$ have been successfully calculated with the DSMC-TSS algorithm. This algorithm is essentially hybrid that employs the two different means to solve the primary and trace species encountered in the RAM C-II test. Such a combination, as shown in the present calculations, provides an effective and efficient means to solve the resolution problem of electron and ions generated due to weakly associative ionization reactions in hypersonic rarefied flows.

\section{REFERENCES}

1. G. A. Bird, Molecular Gas Dynamics and the Direct Simulation of Gas Flows, Oxford: Clarendon Press, 1994.

1. M. S. Ivanov and S. F. Gimelshein, Annu. Rev. Fluid Mech., 30, 469-505 (1998).

3. J. Fan, Adv. Mech., 43,185-201 (2013).

4. G. A. Bird, "Computation of electron density in high altitude re-entry flows", AIAA Paper 89-1882, 1989.

5. W. L. Grantham, "Flight results of a 25,000 foot per second reentry experiment using microwave reflectometers to measure plasma electron density and standoff distance", NASA TN D-6062, 1970.

6. W. L. Jones and A. E. Cross, "Electrostatic measurements of plasma surrounding three 25,000 foot per second reentry flight experiments", NASA SP-252, 1970, pp. 109-136.

7. I. D. Boyd, J. Thermophys. \& Heat Trans., 10, 579-585 (1996).

8. I. D. Boyd, Phys. Fluids, 19, 096102 (2007).

9. J. Fan, Y. H. Zhang, J.Z. Jiang, "An algorithm for predicting trace species in hypersonic rarefied gas flows", CSTAM 2012B03-0174, 2012.

10. K. P. Karipides, I. D. Boyd, and G. E. Caledonia, J. Thermophys. \& Heat Trans., 12, 30-37 (1998).

11. V. K. Dogra, R. J. Collins, and D. A. Levin, AIAA J., 37, 443-452 (1999).

12. S. F. Gimelshein, D. A. Levin, and R. J. Collins, J. Thermophys. \& Heat Trans., 14, 471-479 (2000).

13. M. H. Bortner, "Suggested standard chemical kinetics for flow field calculations - A consensus opinion", in AMRAC Proceedings, vol. 14, Part 1, 1966, pp. 569-581.

14. J. Fan and C. Shen, Acta Aero. Sinica, 13, 405-412 (1995).

15. C. Shen, J. Fan, Z. H. Hu, X. Y. Xu, "A new version of position element algorithm of DSMC in calculating of threedimensional transitional flows", Rarefied Gas Dynamics-20, edited by C. Shen, Peking Univ. Press, Beijing, 1997, pp. 162167.

16. H. L. Liu, J. Fan and C. Shen, "Validation of a hybrid scheme of DSMC in simulating 3-D rarefied gas flows", in Rarefied Gas Dynamics-23, edited by A. D. Ketsdever \& E. P. Muntz, AIP Conference Proceedings 663, American Institute of Physics, Melville, NY, 2002, pp. 382-389.

17. J. Fan, H. L. Liu, J. Z. Jiang, S. L. Peng and C. Shen, Acta Mech. Sinica, 36, 129-139 (2004).

18 C. Park, Nonequilibrium Hypersonic Aerothermodynamics, NewYork: Wiley, 1990. 